\title{
SITUACIÓN DE LA MOTRICIDAD OROFACIAL EN EL CONTEXTO PROFESIONAL DEL TERAPEUTA DE LENGUAJE O FONOAUDIÓLOGO: ACCIONES POLÍTICAS DE IMPLEMENTACIÓN Y LOGROS EN PERÚ ANTONIO MACEDO CORNEJO ${ }^{20}$
}

En el marco de la formación profesional del Terapeuta de Lenguaje o Fonoaudiólogo existen diversos ámbitos de desarrollo profesional, entre ellos, el campo clínico - asistencial, social, empresarial y el de docencia e investigación, es este último el que abre el abanico de oportunidades en relación al empoderamiento de la profesión y del área de la Motricidad Orofacial, toda vez que sin investigación no habría la posibilidad de sistematizar las experiencias recogidas basadas en evidencias, ni mucho menos difundir los resultados y conclusiones de las mismas. Es por ello, que en el contexto de la práctica profesional basada en evidencias se pueden generar propuestas desde el quehacer de la práctica asistencial, así como hacia las políticas publicas dentro de nuestro ámbito de acción, induciendo, así, la demanda de atención, evento que impone el reto de garantizar la oprtunidad y cobertura de servicios con profesionales cada vez más capaces en nuestra tan querida especialidad; por ello, comparo el lugar que tiene la profesión y la especialidad de Motricidad Orofacial en países hermanos y recojo de ellos la posibilidad de empoderarnos más dentro del trabajo interdisciplinario, pongo como ejemplo la iniciativa del Test de la Lengüita, el mismo que fuera trabajado por la Fonoaudióloga Roberta Lopes de Castro Martinelli y posteriormente por su vasta importancia de su trabajo y la voluntad política del estado tuvo la iniciativa de generarse un proyecto de ley sobre el particular el mismo que se formalizo y ofició como una ley, la misma que entró en vigencia desde junio de 2014.

Cosas como esta que pasan fuera de nuestro país hacen que aquí en Perú, soñemos con el empoderamiento de la profesión y luchamos contra la invisibilizacion que se tiene por parte de algunos otros profesionales del equipo multidisciplinario, acciones que muestran en algunos casos a profesionales con un perfil de "todistas" en el ejercicio del quehacer profesional. Por ello, desde la perspectiva del ansiado posicionamiento de la Motricidad Orofacial es que se propuso la constitución de un espacio de desarrollo, de intercambio de ideas, de retroalimentación de conceptos y sobre todo de experiencias, con ello se crea la Comunidad de Motricidad Orofacial Latinoamericana y con este gran paso se procedió a la articulación internacional, a la comúnunión entre profesionales identificados con la carrera y sobre todo con el compromiso no solo a los pacientes, sino a los futuros colegas que están ávidos de más conocimiento, tuvimos la suerte de encontrar en el camino a personalidades de la talla de Irene Marchesan, Licia Paskay, Esther Bianchini, Maria Paz Moya, Norma Chiavarro, Heriberto Rangel, Eliana Rivera, Diana Grandi, Ana Ilse Arraga, Pia Villanueva, Ricardo Santos, Maristella Oncins, Hilton Justino, Lidia D’Agostino, entre otros grandes en su profesión y ahora amigos unidos por la Motricidad Orofacial.

Por cuanto, después de solo corto tiempo, sentimos que el ansiado empoderamiento de la Motricidad Orofacial está asentándose, no solo somos ese espacio de encuentro y de intercambio como empezó esta idea, somos más que eso, producimos material bibliográfico a través de nuestro equipo de profesionales y colaboradores, articulamos en la elaboración de nuevos textos, constituimos un equipo de traducción y con el permiso de sus respectivos autores llevamos el conocimiento en el idioma que corresponda para su entender, por ello reitero, en corto tiempo

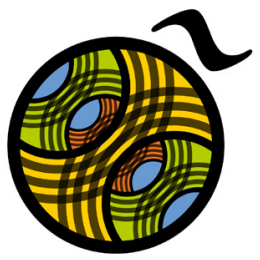

20. Licenciado en Tecnología Médica por la Universidad Nacional Federico Villarreal - UNFV. Especialista en Políticas Públicas e Interculturalidad por la Universidad Nacional Mayor de San Marcos - UNMSM. Maestría en Administración y Gerencia Social. Especialista en Planes Operativo de la Unidad de Servicios de Protección de Personas con Discapacidad - USPPD - INABIF. Gerente Comercial de Red Terapeuta Perú. Docente universitario en Investigación en Ciencias de la Salud, Gerencia de Servicios de Salud, Salud Pública y Rehabilitación Basada en la Comunidad en la Universidad Nacional Federico Villarreal UNFV. Miembro fundador y Director Administrativo de la Comunidad de Motricidad Orofacial Latinoamericana - CMOL. 
hemos avanzado mucho, tenemos el respaldo de organizaciones del más alto nivel de los diferentes países tanto de Europa como de América y nos animamos a realizar eventos de un corte Iberoamericano y con el apoyo de estas organizaciones amigas como la Associação Brasileira de Motricidade Orofacial - ABRAMO, Centro de Especialização em Fonoaudiologia Clinica - CEFAC, la Sociedade Brasileira de Fonoaudiologia - SBFa, la Academy of Orofacial Myofunctional Therapy - AOMT, la Academia of Applied Myofunctional Sciences - AAMS, la Academia Latinoamericana de Disfunciones Estomatognáticas - ALDE, EPAP, la Sociedad Peruana de Ortopedia Funcional de los Maxilares - SPOFM, Instituto de Rehabilitación y Formación en Motricidad Orofacial - IRFMO, entre otras las mismas que avalan nuestros eventos científicos en aras de seguir con este sueño y de dar el empuje al factor docencia que es parte de los objetivos que nos rigen.

Asimismo, contentos de lo logrado y con el ánimo de seguir queriendo cosechar más logros en aras de la profesión, nos hemos propuesto la posibilidad de ser partícipes y actores con protagonismo en la reforma de la salud que se viene dando en nuestro país, queremos proponer iniciativas a manera de proyectos de ley que concluyan justamente en eso, en leyes, en dispositivos legales que permitan en primera instancia una mejor atención a los pacientes, en un segundo lugar, no menos importante que el anterior, la contratación de más profesionales con la especialidad de Motricidad Orofacial para los diferentes centros hospitalarios de la Seguridad Social y del Ministerio de Salud.

Por cuanto, no solo a título personal, sino a nombre del equipo de la Comunidad de Motricidad Orofacial Latinoamericana - CMOL, estamos convencidos que estas ideas que están ya no solo en mente sino en proceso de formulación traerán consigo cambios sustanciales en la profesión y la especialidad y con ello se avecina la reforma que tanto queremos. 